\title{
Recent Advance in Diagnosis, Pathogenesis and Risk Stratification of Essential Thrombocythemia
}

\author{
*Nahla Abdel Moneim Hamed \\ Department of Hematology, Alexandria University, Egypt
}

Submission: January 14, 2017; Published: January 20, 2017

"Corresponding author: Nahla A. M.Hamed, Department of Hematology, Alexandria University, Egypt

\begin{abstract}
In the 2016 version of WHO classification, bone marrow morphology is critical in the distinction between ET and pre-PMF. Reticulin-fiber grading becomes central: grade 1 or less is needed for ET. Furthermore, CALR assessment must be performed in all ET patients without JAK2 mutation. Some prognostic implications have been described for CALR mutations, i.e., a lower risk of thrombosis in ET.

Abbreviations: MPN: Myelo Proliferative Neoplasm; WHO: World Health Organization; JAK: Janus kinase; CALR: Calreticulin; bp: base pair; MPL: Myelo Proliferative Leukemia virus oncogene; TPO: Thrombo Protein; RARS-T: Refractory Anemia with Ring Sideroblasts associated with marked Thrombocytosis; PMF: Primary Myelo Fibrosis; IPSET: The International Prognostic Score for Essential Thrombocythemia; WBC: White Blood Cell; LMWH: Low-Molecular Weight Heparin; SVT: Splanchnic Vein Thrombosis
\end{abstract}

\section{Introduction}

ET is a Philadelphia-negative MPN characterized by sustained clonal proliferation of the megakaryocytic lineage in the bone marrow and an elevated platelet count in the peripheral blood [1]. According to the WHO classifications (especially the 2016 version), diagnostic criteria for ET include: major criteria (i) an elevated platelet count ( $\geq 450 \times 10^{9} / \mathrm{L}$ ); (ii) bone marrow biopsy showing proliferation, mainly of the megakaryocyte lineage, with increased numbers of enlarged, mature megakaryocytes with hyperlobulated nuclei. No significant increase or left shift in neutrophil granulopoiesis or erythropoiesis and very rarely minor (grade 1) increase in reticulin fibers (iii) failure to meet diagnostic criteria for BCR-ABL1 CML, PV, PMF, myelodysplastic syndromes or other myeloid neoplasms; (iv) demonstration of clonal markers, such as JAK2V617F (JAK2), CALR or MPL mutations and a minor criterion: presence of a clonal marker or absence of evidence for reactive thrombocytosis. Diagnosis of ET requires meeting all four of the major criteria above or the first three major criteria and a minor criterion [2]. The incidence rate for ET is $0.2-2.5 / 100.000$ people/year. The prevalence is higher at 38-57/100.000 people, because of the long life expectancy (in excess of 20 years) of ET patients [1].

Pathophysiology: Megakaryocyte progenitor cells in ET are hypersensitive to the action of several cytokines including IL3 and IL6 and possibly TPO. There is controversy regarding spontaneous megakaryocyte formation [3].
Genetic data: Driver mutation (JAK2V617F, CALR and $M P L$ mutations): the frequency of $J A K 2 \mathrm{~V} 617 \mathrm{~F}$ mutation is approximately 50-60\%. Most patients with JAK2-unmutated ET express CALR or MPL mutations, with respective estimated incidences of $25-30 \%$ and 3-5\% [4]. Most CALR mutations were deletions and insertions in exon 9, which cause frame shift mutations. Two CALR mutations are predominantly associated with ET. Type 1 result from a 52-bp deletion, and is found in approximately $50 \%$ of patients and type 2 mutation results from a 5-bp TTGTC insertion, and accounts for approximately $30 \%$ of patients [5].

Approximately $15 \%$ of patients are wild-type for all 3 mutations (i.e., triple-negative)[6]. However, a few triple negative patients carry activating mutation of $M P L$ outside exon 10 [7]. Double-mutant JAK2V617F and CALR positive, patients make a specific subgroup that might be distinct from the JAK2-positive or CALR-positive subgroups and require a careful follow-up and management. The frequency of this cooccurrence was below $1 \%$, in one study [8] and $6.8 \%$ in Lim et al. [9]study. In the last study, these patients had the oldest age, higher thrombotic events and higher major arterial thrombotic events after diagnosis and more patients were in the high-risk group for thrombo hemorrhagic complications [9]. Rashid et al. [10] (2015) reported 55 year old female ET patient who was in complete remission without cytoreductive therapy until their paper publication [10]. 
Non driver mutation: In ET, $46 \%$ of patients had somatic mutations. The most commonly mutated, non driver genes, included DNMT3A (6\%), TET2 (13\%), and ASXL1 (11\%), SF3B1 (5\%), CEBPA (4\%), and TP53, SH2B3, EZH2, and CSF3R (2\% each). Patients with ASXL1, EZH2, SRSF2, or IDH mutations are considered to have a "high molecular risk" profile and were at risk for premature death or leukemic transformation. However, only ASXL1 mutations remained significantly associated with survival [11]. The number of mutations also matters, the presence of 2 or more mutations predicted for worse outcomes; the reported median survival was 12.3 years for patients without a mutation compared with 2.6 years for those with 2 or more mutations. Those with post-ET-MF were more likely to have ASXL1 and EZH2 mutations, compared with those with postPV-MF. The order in which mutations are acquired appears to influence clinical features. It was suggested that those patients who acquired JAK2V617F prior toTET2 are more likely to present with PV than ET [11].

Clinical picture: ET is an indolent myeloproliferative neoplasm [7]. The median age at diagnosis is 60 years. There may be higher prevalence in younger women (approximately 2:1). Many patients are asymptomatic at presentation [3]. The prevalence of constitutional symptoms is relatively high [7]. Patients might also have micro vascular symptoms such as headaches, acroparesthesia, erythromelalgia, peripheral ischemia, transient ischemic attacks, amaurosisfugax or scotom as [1]. Patients may present with splenomegaly (20\%), thrombosis and bleeding. Hemorrhagic event, mostly from GIT is experienced in 13 to $37 \%$ of patients while thromboembolic events in the major vessels or microvasculature are experienced in $22-84 \%$ of patients [3].

Female patients in the child bearing period may experience: live birth rates of $50 \%$ to $70 \%$, and spontaneous abortion rates of $25 \%$ to $50 \%$, mostly during the first trimester. Age, parity, thrombophilia, platelet count, WBC count, and hemoglobin level have not been found to be predictive of pregnancy outcome in ET. Pregnancy complications in women with ET are associated with a higher risk of subsequent thrombosis [7].

Thrombotic risk factors: IPSET is a score which provides prognostic information on risk of thrombosis. It is based on widely accepted risk factors for thrombosis which are age $\geq 60$ years ( 2 points), WBC count $\geq 11 \mathrm{X} 10^{9} / \mathrm{L}$ (1 point), and history of thrombosis (1 point) [7]. JAK2 (V617F) plays a major role in the pathogenesis of thrombosis, whereas CALR or MPL mutation and triple negativity identify patients with lower thrombo embolic risk [7]. Elevated platelet count $\left(>1,000 \times 10^{9} / \mathrm{L}\right)$ was associated with lower arterial thrombotic risk [12].

Comparison of clinical and hematological features of patients with JAK2 mutation and CALR mutation: CALRmutated ET patients had a lower risk of thrombosis than JAK2mutated ET patients [6].Thrombotic events occurred in $26 \%$ of JAK2-mutated ET patients versus $7.7 \%$ of $C A L R$-mutated ET patients. Genetic background such as race may influence the risk of thrombosis [12]. Previous studies have shown higher platelet counts and lower hemoglobin, leukocyte counts and granulocyte counts in CALR mutant compared with JAK2 mutant patients [6]. In addition, platelet counts are higher in type 2 CALR mutant compared with type 1 mutant patients [6]. CALR-mutated ET displaysa phenotype favoring megakaryopoiesis [12].

CALR-mutated ET patients had a longer survival compared with those with $J A K 2 \mathrm{~V} 617 \mathrm{~F}$ mutation. A higher rate of postET-MF transformation was reported in CALR-mutated patients versus JAK2-mutated patients. Interestingly, no CALR-mutant ET patient evolved to PV [11].

Outcome: ET may transform into PV, MF, AML or MDS. However, ET is generally considered a benign illness because it is associated with prolonged survival and a low risk of leukemic transformation [13]. The 15-year cumulative risk of progression to myelofibrosis is about $10 \%$ on average, higher in type 1 CALRmutant than in JAK2-mutant ET while the 15-year cumulative risk of leukemic transformation is about $3 \%$ on average [7].

\section{Diagnostic Consideration}

a) Screening for BCR-ABL1 is important in the diagnostic approach of ET patients to rule out chronic myeloid leukemia and atypical MPN associated with both BCR-ABL1 rearrangement and CALR mutation [7].

b) Assessment of JAK2 mutational status is mandatory in case of unexplained thrombocytosis [14].

c) CALR assessment must be performed in all patients without JAK2 mutations followed by MPL if CALR is absent. These mutations does not replace the need for BM morphologic examination in [1] confirming the diagnosis in triple-negative ET and [2] distinguishing ET from other MPN that share the same mutations, including masked PV and early/ prefibroticmyelo fibrosis [6].

d) JAK2 (V617F) assessment is recommended in patients with SVT because it may be the first marker of a latent MPN. Although, the incidence of CALR exon 9 is low in patients with SVT, CALR assessment can now also be included in the workup of these subjects. Genetic and acquired thrombophilia, in particular the presence of anti-phospholipid syndrome, should be studied in all cases of ET with SVT because ET patients with genetic or acquired thrombophilia are at high risk of recurrent thrombosis [7].

e) At present, there is little evidence to suggest the incorporation of testing for non driver mutation in routine clinical care of ET patients [11].

f) Von Willebr and factor antigen level and ristocetin cofactor activity have to be performed when platelet count is $>1000 \times 10^{9} / \mathrm{L}$ or when an acquired von Willebrand syndrome is anyhow suspected [7]. 
Differential diagnosis: The most common cause of isolated thrombocytosis is a reactive (or secondary)causes [7] due to infections, acute or chronic inflammatory diseases, smoking, iron deficiency, chronic bleeding, postsurgical states, malignancies, hemolysis, rebound after immunosuppressive chemotherapy, and use of drugs (corticosteroids, adrenaline, and TPO mimetics [14].

a. Other clonal myeloid neoplasms [7].

b. Hereditary thrombocytosis and familial ET. Recent reports have described cases of hereditary thrombocytosis associated with non-canonical germ line mutations of JAK2. In familial ET, JAK2 (V617F) is always a somatically acquired event [7].

c. RARS-T, in which JAK2V617F mutational frequency has been reported to be as high as $50 \%$ [6]. Most patients have a combination of SF3B1 mutation (as a driver genetic lesion) and JAK2, MPL, or CALR mutation (as a subclonal genetic lesion); however, up to one-third of patients may have wildtype SF3B1 [7].

d. PV when the hemoglobin/hematocrit level is diagnostically equivocal (as in "masked" PV) [6].

e. Prefibrotic/early prePMF with a high platelet count. Leukocyte count, hemoglobin level, platelet count, serum LDH level, incidence of palpable splenomegaly and circulating CD34 cell count, all are greater in prePMF than ET. In ET, there is no or minor (grade 1) increase of reticulin fibers. The 2016 WHO classification allows the presence of reticulin fibrosis grade $1 \mathrm{in}$ ET, but this is a very rare presentation [14]. On the other hand, age, gender distribution and JAK2V617F mutational frequencies were similar between both groups. The survival rates, leukemic transformation rates, and rates of progression to overt myelofibrosis were significantly worse in prePMF than in ET [15].

\section{Treatment}

Treatment of ET is based on risk stratification: Age $>60$ years, history of vascular complications (thrombosis or major bleeding), and platelet count $\geq 1500 \times 10^{9} / \mathrm{L}$ are the 3 risk factors used to classify patients with ET into low (no risk factors) and high risk (1 or more risk factors). Low-risk ET patients have an incidence of thrombosis similar to that of a healthy control population [7].

The indication for and goals of therapy: are to reduce the risk of life-threatening complications, such as venous or arterial thrombosis or severe bleeding, rather than induce remission or disappearance of the neoplastic clone. Treatment may also help to control vasomotor symptoms [1]. Treatment of ET is based on aspirin in the vast majority of ET cases and cytoreductive therapy [7].

Low-dose aspirin: All ET patients should be managed with low-dose aspirin if micro vascular disturbances are present.
In low-risk ET, anti-platelet therapy reduces the incidence of venous thrombosis in JAK2-mutated patients [7] and the rate of arterial thrombosis in those with cardiovascular risk factors, with no effect on the risk of bleeding. By contrast, in CALRmutated patients, anti-platelet therapy did not affect the risk of thrombosis whereas it was associated with a higher incidence of bleeding. In high-risk ET patients, anti-platelet therapy should be administered to all patients except those with extreme thrombocytosis (>1500 x $10^{9} / \mathrm{L}$ ) [7].

Cytoreductive therapy: Extreme thrombocytosis is frequently associated with acquired von Willebrand syndrome [7]. So, A platelet count $>1500 \times 10^{9} / \mathrm{L}$ represents an indication to cytoreductive treatment [7] with hydroxyurea, or interferon, or an agrelide when indicated or ruxolitinib when inadequate response to hydroxyurea occurs [14].

Treatment of pregnant females: Aspirin is safe for both mother and fetus. It is recommended for all pregnant women with ET, unless contraindicated. Low-dose aspirin is effective in preventing preeclampsia [7]. LMWH is added to lowdose aspirin in case of previous major thrombosis or severe pregnancy complication. Consider interferon $\alpha$ if the platelet count is $>1500 \times 109 / \mathrm{L}$ or in case of previous major bleeding. The optimal time to discontinue anti-platelet treatment is generally about 2 weeks before delivery for any possible instrumental delivery and epidural or spinal anesthesia. After delivery, treat all women with ET with LMWH for 6 weeks to prevent deep vein thrombosis [7].

Cytoreductive therapy and natural history of disease: Hydroxyurea does not specifically target the mutant clone and is therefore unlikely to modify the natural history of disease. Pegylated interferon $\alpha-2$ a has been shown to induce sustained complete molecular response in a subset of patients with JAK2 (V617F)-mutant ET. In addition, recent reports describe the positive effect of interferon $\alpha$ in patients with CALR-mutant ET [7]. Those with additional mutations had poorer molecular responses compared with those with CALR alone. Analyses of mutation type also suggested a differential effect from IFN- $\alpha$ on mutated clones, because some patients experienced a decrease in the $C A L R$ burden but an increase in the clonal burden of other somatic mutations [11]. These observations do not necessarily mean that interferon $\alpha$ can modify the natural history of disease but they at least indicate that this drug can target the myelo proliferative clone [7].

Future therapy: Whether JAK inhibitors can provide beneficial effects in patients with ET remains to be established in ad hoc clinical trials, but ruxolitinib has already proved to be effective in patients with a relatively aggressive disease [7]. A novel ET treatment concept involves telomerase inhibition. Response was seen regardless of the mutational profile, although more pronounced in those with JAK2 mutation. Mutant allele burdens were also reduced by $15 \%$ to $66 \%$ in those with MPL or CALR mutations [11]. 


\section{Cancer Therapy \& Oncology International Journal}

\section{Conclusion}

There is a tremendous scientific advance in the last decade in diagnostic capability, and disease pathogenesis of essential thrombocythemia. However still there are unanswered questions especially as regards progression of disease.

\section{References}

1. Falchi L, Bose P, Newberry KJ, Verstovsek S (2016) Approach to patients with essential thrombocythemia and very high platelet counts: what is the evidence for treatment? Br J Haematol.

2. Arber DA, Orazi A, Hasserjian R, Thiele J, Borowitz MJ, et al. (2016) The updated WHO classification of hematological malignancies. The 2016 revision to the World Health Organization classification of myeloid neoplasms and acute leukemia. Blood 127(20): 2391-2405.

3. Aaron T Gerds (2016) Myeloproliferative neoplasm.

4. Jacob Grinfeld, Jyoti Nangalia, Anthony R Green (2017) Molecular determinants of pathogenesis and clinical phenotype in myeloproliferative neoplasms. Haematologica 102(1): 7-17.

5. Kim SY, Im K, Park SN, Kwon J, Kim J-A, et al. (2015) CALR, JAK2, and MPL Mutation profiles in patients with four different subtypes of myeloproliferative neoplasms primary myelofibrosis, essential thrombocythemia, polycythemia vera, and myeloproliferative neoplasm, unclassifiable. Am J Clin Pathol 143(5): 635-644.

6. Tefferi A, Barbui $T$ (2015) Essential thrombocythemia and polycythemia vera: focus on clinical practice. Mayo Clin Proc 90(9): 1283-1293.

7. Rumi E, Cazzola M (2016) How I treat essential thrombocythemia. Blood 128 (20): 2403-2416.
8. Ahmed RZ, Rashid M, Ahmed N, Nadeem M, Shamsi TS (2016) Coexisting JAK2V617F and CALR exon 9 mutations in myeloproliferative neoplasms - do they designate a new subtype? Asian Pac J Cancer Prev 17(3): 923-926.

9. Lim KH, Chang YC, Gon-Shen CC, Lin HC, Wang WT, et al. (2015) Frequent CALR exon 9 alterations in JAK2V617F-mutated essential thrombocythemia detected by high-resolution melting analysis. Blood Cancer J 5: 295.

10. Rashid M, Ahmed RZ, Ahmed S, Nadeem M, Ahmed N et al. (2016) Coexisting JAK2V617F and CALR exon 9 mutation in essential thrombocythemia. Indian J Hematol Blood Transfus 32(S1): 112-116.

11. Shammo JM, Stein BL (2016) Mutations in MPNs: prognostic implications, window to biology, and impact on treatment decisions. Hematology Am Soc Hematol Educ Program 2016(1): 552-560.

12. Kubuki Y, Shide K, Kameda T, Yamaji T, Sekine M, et al. (2017) Differences in hematological and clinical features between essential thrombocythemia cases with JAK2- or CALR-Mutations. Ann Lab Med 37(2): 159-161.

13. Chim CS, Kwong YL, Lie AK, Ma SK, Chan CC, et al. (2005) Long-term outcome of 231 patients with essential thrombocythemia prognostic factors for thrombosis, bleeding, myelofibrosis, and leukemia. Arch Intern Med 165: 2651-2658.

14. Passamonti F, Maffioli M (2016) Update from the latest WHO classification of MPNs: a user's manual. Hematology Am Soc Hematol Educ Program 2016(1): 534-542.

15. Barbui T, Thiele J, Vannucchi AM, Tefferi A (2016) Myeloproliferative neoplasms: morphology and clinical practice. Am J Hematol 91(4): 430-433.

\section{Your next submission with Juniper Publishers will reach you the below assets}

- Quality Editorial service

- Swift Peer Review

- Reprints availability

- E-prints Service

- Manuscript Podcast for convenient understanding

- Global attainment for your research

- Manuscript accessibility in different formats

( Pdf, E-pub, Full Text, Audio)

- Unceasing customer service

Track the below URL for one-step submission https://juniperpublishers.com/online-submission.php 